MCCLoy, E. W. (1958). J. gen. Microbiol. 18, 198-220

\title{
Lysogenicity and Immunity to Bacillus Phage W
}

\author{
By ELINOR W. MCCLOY \\ Department of Bacteriology, London School of Hygiene and Tropical Medicine, \\ Keppel Street, London W.C. 1
}

SUMMARY: Cultures of strain W of Bacillus cereus sometimes contained a phage, $W \alpha$, which could form plaques on strain $W$ itself. Phage $W \alpha$ was found to be a virulent mutant of a temperate phage, $W \beta$, with which strain $W$ was lysogenic. Another non-lysogenizing mutant, $\mathbf{W} \gamma$, was also isolated which, unlike $\alpha$, did not form plaques on strain $W$. The genetic determinants of $\alpha, \beta$ and $\gamma$ are probably allelic. Neither phage $\gamma$ nor phage $\beta$ formed plaques on bacterial strains lysogenic with $\beta$, but these strains were not completely immune, even to the temperate phage. This finding shows that lysogenicity does not invariably confer complete immunity to the carried phage. The colonial appearance of a strain was altered after lysogenization in a way suggesting that the alteration was due to its incomplete immunity to the temperate phage produced during growth.

Lysogenic strains of bacteria are usually immune to the phage they produce and the immunity is conferred on them by the prophage (Lwoff, 1953; Bertani, 1958). The phages produced by lysogenic strains are temperate (by definition capable of lysogenizing), but temperate phages can give rise by mutation to virulent phages (by definition incapable of lysogenizing; Jacob, Lwoff, Siminovitch \& Wollman, 1953). In general, lysogenic strains are immune to the commoner virulent mutants as well as to the temperate phage, and thus do not manifest any cultural abnormality to suggest that they produce a phage. A strain of Bacillus cereus, named W, attracted attention because platings from old broth cultures yielded bacterial growth which was interrupted by patches of partial clearing. A phage was isolated from these patches which produced plaques on strain W. It was easily shown that the cultures were not merely cultures contaminated with phage, and thus it appeared that strain $W$ produced a phage to which it was itself susceptible. The most probable explanation for the phenomenon was that the phage was a mutant of a temperate phage with which strain $W$ was lysogenic. The phage, which has been called phage $W \alpha$, also attacked strains of Bacillus anthracis and a small proportion of strains of $\boldsymbol{B}$. cereus (McCloy, 1951). It did not lysogenize sensitive strains. Subsequently, other phages were isolated from B. cereus strain W which did not form plaques on it. Filtrates of cultures of strain $W$ of any age contained a phage, designated $\mathbf{W} \beta$, which attacked all strains which were sensitive to phage $\alpha$ except strain $W$. Phage $\beta$ could lysogenize, and the lysogenic derivatives of sensitive bacterial strains were, like strain $W$, grossly susceptible to phage $\alpha$ but not to phage $\beta$. A third phage, named $\mathbf{W} \gamma$, found in exponentially growing cultures of strain $\mathbf{W}$, did not lysogenize, and attacked strains sensitive to phage $\beta$. Phage $\gamma$ did not form plaques on strains lysogenized by $\beta$. Bacteria artificially lysogenized by phage $\beta$ gave rise 
spontaneously to phages which resembled $\alpha$ and $\gamma$ in all characters tested, and which were therefore assumed to be the same as the phages $\alpha$ and $\gamma$ directly isolated from strain $\mathbf{W}$.

The three phages $\alpha, \beta$ and $\gamma$ are identical in serological and other characters (McCloy, to be published). The present paper discusses the relationship between them, and their behaviour in relation to the phenomena of lysogenicity and the immunity which it confers. Phage $\beta$ had the characters of a temperate, that is a potentially lysogenizing, phage, and phages $\alpha$ and $\gamma$ were virulent, that is unable to lysogenize. Phage $\alpha$ was differentiated from phage $\gamma$ by its ability to form plaques on the lysogenic strains; as will be seen, however, phages $\gamma$ and $\beta$ were not quite without action on the lysogenic strains.

All single colony isolates of Bacillus cereus strain $\mathbf{W}$ which were tested yielded the three phages, $\alpha, \beta$ and $\gamma$. Hence, each cell of strain $W$ was presumed to carry the precursor of each phage. Since lysogenization of sensitive strains with phage $\beta$ always yielded cultures from which phages $\alpha$ and $\gamma$ could be obtained, the three phages evidently had a common genetic origin; this conclusion was also supported by their identity in serological and other characters. The precursor in the naturally lysogenic strain $W$ was probably $\beta$ because, of the three, $\beta$ was the only temperate phage in relation to the sensitive indicator strains used, and because in strain $W$, as in the strains artificially lysogenized with $\beta$, phage $\beta$ was produced regularly, whereas $\alpha$ and $\gamma$ appeared more rarely, as if their appearance were a consequence of mutation.

\section{ME'THODS}

Bacteria. The lysogenic strain of Bacillus cereus, strain W, had the characters of the species B. cereus (Smith, Gordon \& Clark, 1952; Knight \& Proom, 1950; Chu, 1949 and personal communication). The strain used in most of the experiments as indicator and propagating strain for the phage produced by strain $\mathbf{W}$ was an atypical strain of $B$. anthracis, strain Davis, since confluent growth of this strain was very much smoother than that of typical strains of B. anthracis, and plaques were easily seen in lawns produced either by flooding plates with bacterial suspensions or by the agar layer method. Strain Davis was identified as a strain of $\boldsymbol{B}$. anthracis by $\mathrm{Dr} \mathbf{H}$. P. Chu (personal communication), but it was atypical in that it was of low virulence for mice, did not form spores, and its cells were less than $0.9 \mu$. in diameter. It grew in broth as a mixture of short chains and filaments, giving a uniform turbidity. No means were found of making it grow in elements more uniform in length. It formed small colonies of rather rough appearance on nutrient agar incubated in air (Pl. 1, fig. 3), but the growth could be emulsified fairly easily, in contrast to the growth of typical $B$. anthracis. It had retained the ability to form capsules, for it formed mucoid colonies on serum agar incubated in air with added $\mathrm{CO}_{2}$ (Sterne, 1937; Chu, 1952). Other strains used as indicators and propagating strains for the phage were an avirulent asporogenous strain of $\boldsymbol{B}$. anthracis called Lindsay, which formed almost smooth colonies, and three typical virulent strains of $B$. anthracis, strains 2160,1444 and $\mathrm{H}_{3}$. Variants of strain 2160 
which formed colonies smoother than those of the parent strain, and which were asporogenous, were isolated by incubating for some days cultures of strain 2160 in $100 \mathrm{ml}$. volumes of broth to which $0.025 \%(\mathrm{w} / \mathrm{v}) \mathrm{CaCl}_{2}$ had been added (Bordet \& Reneaux, 1930; Reneaux, 1952); one such variant, called $2160 \mathrm{~S}$, was used in some experiments. One strain of B. cereus, NCTC 1651, which was sensitive to the phage, was also used. None of the strains of $B$. anthracis could be shown to be lysogenic. Usually only the strain names will be used in this paper except where the species (anthracis or cereus) of the strain is relevant to the subject.

Measurements of bacterial turbidity were made with an EEL nephelometer (Evans Electroselenium Ltd, Harlow, Essex).

Media. The fluid media used in most of the experiments were Hartley tryptic digest broth, and peptone meat-extract (Lemco) broth. The solid medium consisted (as \%, w/v) of: 1, peptone (Evans); 1, meat extract (Lemco); 1, NaCl; 1.2, New Zealand agar (Davis), and was supplemented with $0.5 \%$ $(\mathrm{w} / \mathrm{v})$ liver extract (Oxoid). On this medium without the liver-extract supplements, lawns of strain Davis were thin, and the plaques produced by the temperate phage $\beta$ were clear and could not be distinguished from those produced by phages $\alpha$ and $\gamma$. It was found, however, that addition of liver extract gave thicker lawns, and that the plaques of phage $\beta$ then contained bacterial growth and could now easily be distinguished from plaques of phages $\alpha$ and $\gamma$ which were still clear.

Phage stocks. These were made by growing the phages on sensitive strains in broth. Stocks of the phages grown on strain Davis were also made by the agar layer method (Swanstrom \& Adams, 1951), since by this method higher titres were obtained. Lysates were filtered through sintered-glass or Chamberland filters. With preparations grown on strain Davis, titres of $1 \times 10^{9}$ to $5 \times 10^{9}$ particles $/ \mathrm{ml}$. were obtained by the broth method, and titres of $1 \times 10^{10}$ to $5 \times 10^{10}$ particles $/ \mathrm{ml}$., and occasionally as high as $2 \times 10^{11}$ particles $/ \mathrm{ml}$. were obtained with phages $\alpha$ and $\gamma$ by the agar layer method.

Artificially lysogenic strains. These were made by exposing sensitive strains to the phage on solid medium; the resulting bacterial growth was subcultured, and an isolated colony entire in outline was tested for the presence of phage. Three or four successive single-colony isolations were made from satisfactory colonies before the bacterial strain was used. Lysogenic strains have been named in the conventional way with the name of the phage in brackets following the name of the bacterial strain, for example Davis $(\beta)$.

Phage $\beta$ LC. In the earlier experiments, bacterial strains lysogenic with $\beta$ were the only available differential indicators for phage $\alpha$, and they were unsatisfactory indicators in that they themselves could produce phage $\alpha$, and fairly frequently showed spontaneous phage $\alpha$ plaques. However, in the course of the work, a variant of phage $\beta$ was obtained which was not observed to give rise by mutation to phage $\alpha$; with this phage very much more satisfactory differential indicator strains could be made for detection and titration of phage $\alpha$. This variant phage, named $\beta \mathrm{LC}$, was derived as a spontaneous mutant of a plaque-type mutant arising when irradiated phage $\beta$ was plated with irradiated 
bacteria of strain Davis (Weigle, 1953). The genetic differences between phage $\beta$ and phage $\beta \mathrm{LC}$ have not been investigated, but phage $\beta \mathrm{LC}$ evidently differs from phage $\alpha$ at more than one locus, for mixed infection of strain Davis with phage $\alpha$ and phage $\beta$ LC yielded a few particles of phage $\beta$.

Titrations of phage. Titrations of phage on non-lysogenic strains, and on strains lysogenic with phage $\beta \mathrm{LC}$ were, in general, made by the agar layer method (Hershey, Kalmanson \& Bronfenbrenner, 1943). The agar layer method could not be used for titration of phage $\alpha$ on bacterial strains lysogenic with $\beta$ because of the frequency with which these strains themselves produced phage $\alpha$; with these strains, a modified Miles \& Misra method was used (Miles \& Misra, 1938), the areas of the plate which had not been inoculated with phage acting as a control for spontaneous plaques. In the modified Miles \& Misra method, well-dried agar plates were sown for confluent lawns by flooding with suspensions of bacteria, the excess being pipetted off. When the surface had dried, $\mathbf{0 . 0 2} \mathrm{ml}$. drops of phage suspension were spotted on marked areas. With both methods, the plaques were counted after the plates had been incubated at $37^{\circ}$ for $24 \mathrm{hr}$. The efficiency of plating of the phages on nonlysogenic strains was the same by the two methods of titration. Except where otherwise stated, the phage titres given are the concentrations of plaque-forming particles on non-lysogenic strain Davis.

For assaying free phage in the presence of lysogenic or infected bacteria, streptomycin-resistant variants of strain Davis or strain $2160 \mathrm{~S}$, or their derivatives lysogenic with $\beta \mathrm{LC}$, were used as indicators, streptomycin being added to the agar medium to a concentration of $100 \mu \mathrm{g} / \mathrm{ml}$. (Bertani, 1951). The efficiency of plating of free phage on the streptomycin-resistant variants on streptomycin agar was the same as on the streptomycin-sensitive strains on agar without streptomycin. Plaques were not produced on streptomycin-resistant indicators on streptomycin agar by infected streptomycin-sensitive bacteria early in the latent period, but it seemed possible that they might be produced not only by free phage, but also by some, or perhaps all, the infected bacteria late in the latent period, when the bacteria contained mature phage particles. The minimum latent period of phage $\gamma$ in strain Davis was about 65-70 min. and control experiments in which infected bacteria were passed through anti-phage serum to neutralize any phage released early showed that $60 \mathrm{~min}$. after infection about half the infected bacteria were able to produce plaques on streptomycin agar.

Anti-phage sera. These were produced by intravenous inoculation of rabbits with phage grown on strain Davis. The rabbits received weekly inoculations of $1 \mathrm{ml}$. of broth culture filtrates of titre about $5 \times 10^{9}$ particles $/ \mathrm{ml}$. for 6 weeks or more.

\section{RESULTS}

Temperate and virulent characters of the phages

Neither phage $\alpha$ nor phage $\gamma$ established lysogenicity; secondary growth arising when sensitive strains were lysed by these phages was never found to be lysogenized by them. Resistant growth owed its resistance to failure to absorb 
the phage. Lysogenic bacteria producing phage $\beta$ or phage $\beta \mathrm{LC}$ could be obtained when sensitive strains were exposed to phage $\beta$ or phage $\beta \mathrm{LC}$ respectively. These absorbed the phage as readily as the parent non-lysogenic strains.

Phages $\alpha$ and $\gamma$ formed clear plaques indistinguishable from each other on non-lysogenic strain Davis (Pl. 1, fig. 1). Although phage $\beta$ formed clear plaques on strain Davis on ordinary nutrient agar, it formed plaques containing bacterial growth on agar supplemented with liver extract. The plaques of phage $\beta$ on liver-extract agar were target-like in appearance, each having a clear centre surrounded by a ring of bacterial growth separated from the main lawn by a narrow line of clearing ( $\mathrm{Pl}$. 1, fig. 1). Of various other supplements tried (yeast extract, various amino acids, purines, nicotinamide, $\mathrm{MnCl}_{2}$, $\mathrm{MgSO}_{4}, \mathrm{FeSO}_{4}, \mathrm{ZnSO}_{4}, \mathrm{CoCl}_{2}, \mathrm{CaCl}_{2}, \mathrm{KCl}, \mathrm{Na}$ citrate, $\mathrm{Na}$ oxalate) only manganese, known to influence the metabolism of Bacillus spp. (Weinberg, 1955), permitted the formation of target plaques by phage $\beta ; 2 \times 10^{-6} \mathrm{M}$ $\mathrm{MnCl}_{2}$ was sufficient with one batch of Lemco agar. Addition of manganese also promoted thicker growth of the lawns of strain Davis.

The plaques of phage $\beta \mathbf{L C}$ on strain Davis had a central area of thick growth surrounded by a fairly wide clear rim (Pl. 1, fig. 2).

\section{Characters of bacterial strains artificially lysogenic with phage $\beta$}

Lysogenic derivatives of seven bacterial strains were examined. These were the atypical Bacillus anthracis strains, Davis, Lindsay and $2160 \mathrm{~S}$; the typical $B$. anthracis strains, 2160,1444 and $\mathrm{H}_{3}$; and $B$. cereus 1651 . All the lysogenic derivatives of these strains resembled the naturally lysogenic $B$. cereus strain $\mathbf{W}$ in that phage $\alpha$, but not phage $\gamma$ or phage $\beta$, formed plaques on them, and in that they regularly yielded phage $\beta$ and sometimes phage $\gamma$ and phage $\alpha$. Phage $\alpha$ was found in broth cultures incubated for several days and, in addition, a few phage $\alpha$ plaques sometimes appeared when the bacterial strains were grown on solid medium. With the artificially lysogenic strains as well as with the naturally lysogenic strain $\mathrm{W}$, these spontaneous plaques were much more frequent on nutrient agar supplemented with liver extract than on nutrient agar without this supplement. The phage was adsorbed rapidly to the atypical strains of $B$. anthracis, and slowly to the typical strains and to $B$. cereus strains 1651 and $W$. Spontaneous phage $\alpha$ plaques were much more frequently seen with the atypical strains of $B$. anthracis than with the others.

Strain Davis. Stable lysogenic bacteria of this strain could be obtained by subculture either of the ring of growth in the plaques of phage $\beta$ or of the secondary growth in broth cultures cleared by phage $\beta$. The first subculture from either source was eroded, suggesting that the rate of stable lysogenization was low. Boyd (1951) found that with the group A phages of Salmonella typhimurium, a high phage: bacterium ratio increased the rate of lysogenization sufficiently to prevent clearing of broth cultures, but with phage $\beta$ no such effect was seen.

The appearance of the growth of all of about 30 isolates of strain Davis $(\beta)$ differed markedly from that of non-lysogenic strain Davis (Pl. 1, figs 3,4 ). The confluent growth was thinner and sometimes showed vitreous patches of partial 
clearing; the isolated colonies were also thinner and usually had a central spot of partial clearing. These areas of partial clearing were not caused by phage $\alpha$ spontaneously appearing.

Strain Davis lysogenic with $\beta$ contrasted markedly with strain Davis lysogenic with $\beta$ LC. Phage $\beta$ LC evidently established stable lysogenicity more readily than phage $\beta$, for the first subculture from plaques of phage $\beta \mathrm{LC}$ did not give eroded growth, and lysogenicity with $\beta \mathrm{LC}$ did not alter the cultural characteristics of strain Davis (Pl. 1, fig. 5). A further difference was observed
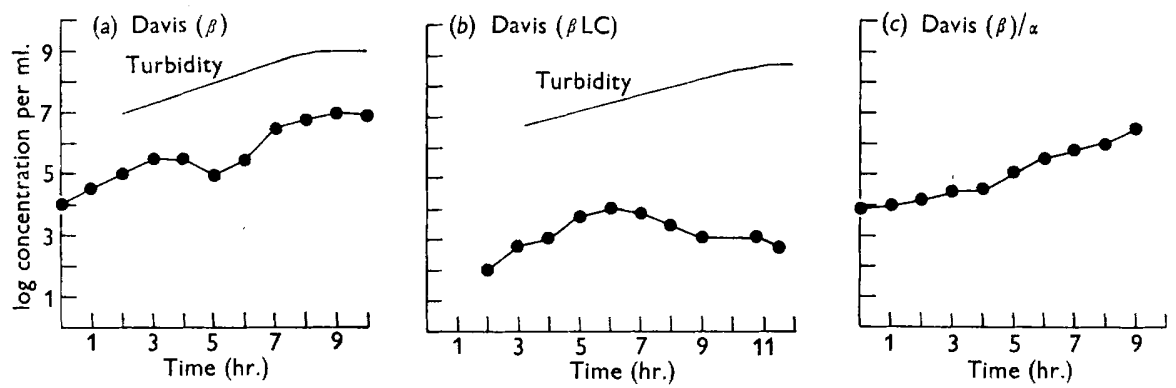

Fig. 1. Spontaneous production of phage in broth cultures of strains Davis $(\beta)$, Davis $(\beta \mathrm{LC})$ and a variant of Davis $(\beta)$ not absorbing phage $\beta$. Cultures of strains Davis $(\beta)$, Davis $(\beta L C)$ and a variant of strain Davis $(\beta)$, Davis $(\beta) / \alpha$, not absorbing phage $\beta$, in $50 \mathrm{ml}$. volumes of broth in $250 \mathrm{ml}$. conical flasks were incubated at $37^{\circ}$. The inoculum in each case was $0.5 \mathrm{ml}$. of an $18 \mathrm{hr}$. broth culture. Phage production was followed by plating samples with streptomycin-resistant strain Davis on streptomycin agar. Bacterial growth of strains Davis $(\beta)$ and Davis $(\beta \mathbf{L C})$ was followed nephelometrically; the positions of the growth curves are only approximate and were estimated from comparisons with turbidities of cultures of Escherichia coli on which viable bacterial counts were made. The growth curve of the non-absorbing variant could not be measured either by nephelometry or colony counts, for it grew in long chains which formed a tangled mass at the bottom of the flask. As far as it could be judged by eye, the culture grew at about the same rate as the cultures of strains Davis $(\beta)$ and Davis ( $\beta$ LC).

when the production of phage in broth cultures of the two lysogenic derivatives was compared. At comparable bacterial densities, very much more phage was present in cultures of strain Davis $(\beta)$ than in cultures of strain Davis $(\beta \mathrm{LC})$, and the curves of phage production by the two strains were different; one experiment is illustrated in Fig. $1 a, b$. The concentration of free phage in cultures of either strain rose steadily until a certain bacterial density was reached, after which it fell, presumably because the rate at which phage was adsorbed to bacteria now became greater than the rate at which it was liberated. As cultures of strain Davis $(\beta \mathrm{LC})$ continued to grow, the fall in free phage concentration continued, but with cultures of strain Davis $(\beta)$, a second rise in phage concentration occurred. Since phage was evidently reabsorbed, the second rise with strain Davis $(\beta)$ might have been the result of phage multiplication caused by superinfection of the lysogenic bacteria. Experiments were therefore made to test the effect of superinfection of lysogenic strain Davis with phage $\beta$. These experiments (described later) showed that superinfection of strain Davis $(\beta)$ with phage $\beta$ did in fact cause increased amounts of phage to be produced, and that phage $\beta$ had some ability to induce its corresponding prophage. 
A variant of strain Davis $(\beta)$ which did not absorb phage $\beta$ was selected by exposing strain Davis $(\beta)$ to phage $\alpha$. The concentration of free phage increased steadily in cultures of this variant (Fig. 1c), supporting the idea that the second rise seen in cultures of phage-absorbing strain Davis $(\beta)$ was due to induction caused by superinfection and not to spontaneous induction happening more often in the later stages of growth.

Alteration of the colonial appearance of a strain with lysogenicity has been reported by Ionesco (1953). Strain Davis $(\beta)$ was evidently not completely immune to phage $\beta$. It thus seems possible that no further explanation is needed for the altered cultural appearance of strain Davis $(\beta)$ (thinner growth and hollowed centres of the colonies) than the fact that this lysogenic strain can undergo what is virtually an autolysis by producing phage to which it is itself susceptible.

Strains other than strain Davis. All of 3 separate isolates of lysogenic Bacillus anthracis strain $\mathrm{H}_{3}, 5$ separate isolates of lysogenic B. anthracis strain 1444 and 2 separate isolates of lysogenic $B$. cereus strain 1651 , resembled their parent strains in colonial morphology and in forming spores. Four out of 5 separate isolates of lysogenic $B$. anthracis strain 2160 also resembled the parent strain in these characters; the fifth isolate, however, formed smoother colonies, was asporogenous and absorbed the phage rapidly, in contrast to the parent strain and its other lysogenic derivatives. This exceptional isolate of lysogenic strain 2160, which has been called $2160(\beta) \mathrm{S}$, was picked because of its aberrant colonial appearance, from the secondary growth on a plate on which strain 2160 had been exposed to phage $\beta$. Since the other isolates of strain $2160(\beta)$ resembled the parent strain, it seems unlikely that the altered characters of this isolate were due to its lysogenicity. Efforts to rid it of its lysogenicity failed, but it seems probable that it was a smooth variant of strain 2160 which had been lysogenized, for variants of non-lysogenic strain 2160 resembling it in colonial morphology, rapid adsorption of phage and failure to spore were obtained by incubating strain 2160 at $37^{\circ}$ for 7 days in broth containing $0.025 \%$, w/v, $\mathrm{CaCl}_{2}$ (Bordet \& Renaux, 1930; Renaux, 1952). These variants $(2160 \mathrm{~S})$ were not altered in their colonial characteristics by lysogenization. Non-lysogenic B. anthracis strain Lindsay formed smooth colonies and was asporogenous; lysogenization did not alter its colonial characteristics.

Phage $\beta$ adsorbed rapidly to the atypical Bacillus anthracis strains Davis, $2160 \mathrm{~S}$ and Lindsay, but exceedingly slowly to the typical $B$. anthracis strains $\mathrm{H}_{3}, 1444$, and 2160 and to $B$. cereus 1651 . Slow adsorption might have accounted for the failure of lysogenicity with phage $\beta$ to alter the colonial appearance of these latter strains, but could not account for its failure to alter the appearance of strains $2160 \mathrm{~S}$ and Lindsay. The growth of these two strains was more luxuriant than that of strain Davis, and so perhaps any effect on their colonial appearance caused by lysis through superinfection was counteracted by the more luxuriant bacterial growth.

Attempts to induce lysogenic strains with ultraviolet radiation and nitrogen mustard. Attempts were made to induce the naturally lysogenic strain $\mathrm{W}$ and the artificially lysogenic strain Davis $(\beta)$ with ultraviolet radiation. It was 
clear that prophage $\beta$ was inducible to some extent, for an increase of phage up to 40 -fold of that in a control unirradiated culture was obtained on several occasions with strain $\mathbf{W}$, and a small increase was also obtained with Davis $(\beta)$. However, although a number of different conditions of cultivation were tried with strain $W$, and bacteria at different stages of their growth were tested, satisfactory induction was never obtained; attempts to induce strain $\mathbf{W}$ with nitrogen mustard failed.

\section{Ability of phage $\alpha$ to attack lysogenic strains}

Lysogenicity with phages $\beta$ or $\beta$ LC conferred little immunity against phage $\alpha$; phage $\alpha$ formed plaques on the naturally lysogenic Bacillus cereus strain $W$ and on the artificially lysogenic derivatives of sensitive $B$. anthracis and $B$. cereus strains, and its efficiency of plating on the lysogenic derivatives of strains
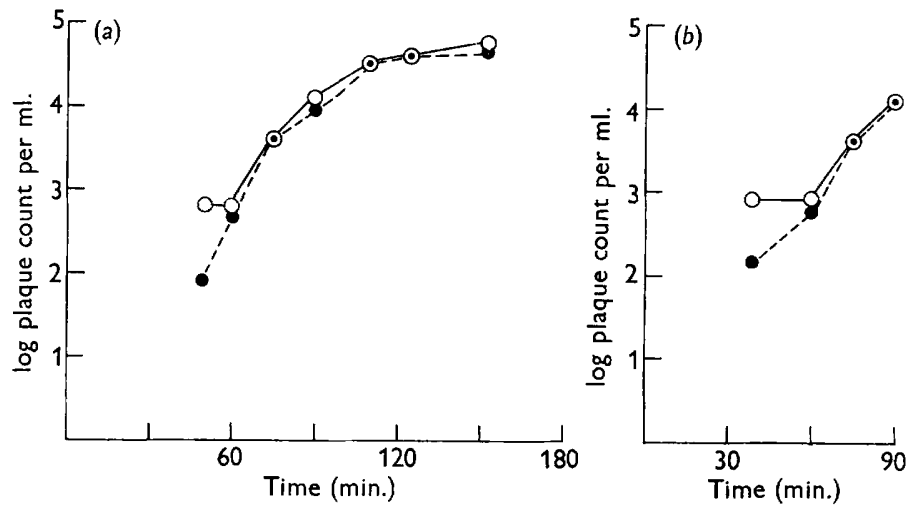

Fig. 2. Growth curves of phage $\alpha$ in strain Davis $(\beta L C)$ and non-lysogenic strain Davis. Phage $\alpha$ at a concentration of $9 \times 10^{6}$ particles $/ \mathrm{ml}$. was mixed at $37^{\circ}$ with broth suspensions of strain Davis $(\beta \mathrm{LC})$ and non-lysogenic strain Davis of turbidity approximately corresponding to that of $5 \times 10^{8}$ Escherichia coli $/ \mathrm{ml}$. After $10 \mathrm{~min}$. for adsorption, the mixtures were treated with anti-phage serum and equally diluted into broth at $37^{\circ}$. Samples taken at intervals were plated with strain Davis $(\beta \mathbf{L C})$ on agar without streptomycin and with streptomycin-resistant strain Davis $(\beta \mathbf{L C})$ on streptomycin agar. $(a)=$ growth curve in strain Davis $(\beta \mathrm{LC}) . \quad(b)=$ growth curve in non-lysogenic strain Davis. $O=$ plaques on agar without streptomycin. $O=$ plaques on streptomycin agar.

Davis and 2160S was about half that on the non-lysogenic parent strains. In one-step growth experiments, phage $\alpha$ had the same minimum latent period in strain Davis $(\beta)$ and strain Davis $(\beta \mathrm{LC})$ as in non-lysogenic Davis, and showed the same curve of increase of plaque-forming particles. Fig. 2 shows the results of an experiment in which growth of phage $\alpha$ in strain Davis $(\beta L C)$ with low multiplicity of infection was followed for $155 \mathrm{~min}$. and compared with growth in non-lysogenic strain Davis up to 90 min. Samples from the infected cultures were plated with streptomycin-resistant strain Davis $(\beta \mathrm{LC})$ on streptomycin agar as well as with strain Davis $(\beta \mathrm{LC})$ on agar without streptomycin. As mentioned previously (Methods), not only free phage but also infected streptomycin-sensitive bacteria once they contain mature phage produce plaques on 
streptomycin agar. In the present experiment, the ratio of plaques on streptomycin agar to plaques on agar without streptomycin at $40 \mathrm{~min}$. was the same with both hosts, and it may be inferred that the same proportion of infected bacteria of both strains contained mature phage by this time. These findings suggest that phage $\alpha$ started to multiply as soon in lysogenic as in nonlysogenic strain Davis.

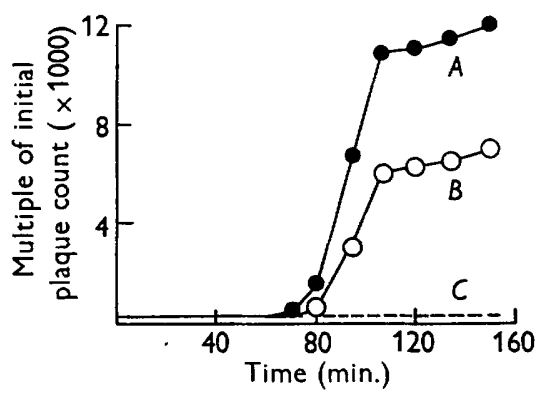

Fig. 3

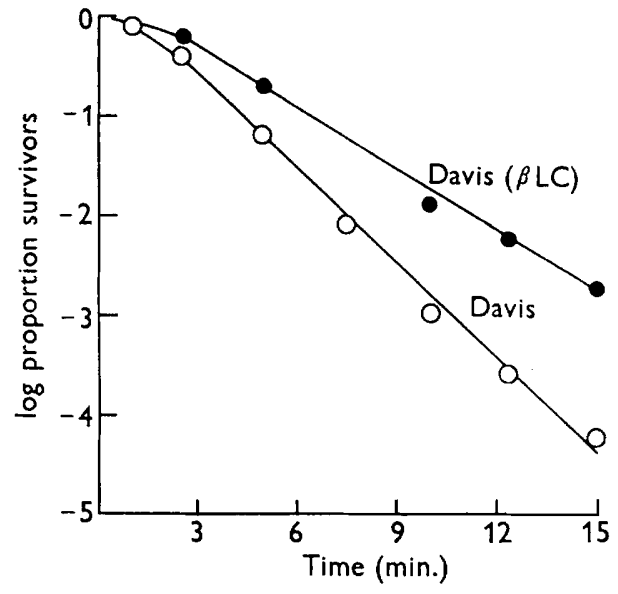

Fig. 4

Fig. 3. Growth curve of phage $\alpha$ in strain Davis $(\beta)$. A suspension of strain Davis $(\beta)$ was divided into two parts, phage $\alpha$ was added to one and, after $3 \mathrm{~min}$. for its adsorption, both parts were treated with anti-phage serum to neutralize the free phage present. The multiplicity of infection with phage $\alpha$ was $9 \cdot 8 /$ chain of bacteria. Suitable dilutions of both suspensions in broth were incubated at $37^{\circ}$, and samples taken from them at intervals were plated with non-lysogenic strain Davis on agar without streptomycin. The plaque counts expressed as multiples of the initial number at $15 \mathrm{~min}$. are plotted against time after the adsorption mixture was made. Curve $A$ shows the numbers of phage $\alpha$ plaques and curve $B$ the numbers of phage $\beta$ plaques produced by the culture superinfected with phage $\alpha$. Curve $C$ shows the numbers of plaques (all $\beta$ ) produced by the control culture without phage $\alpha$.

Fig. 4. Restoration of ultraviolet-inactivated phage $\alpha$ in strain Davis $(\beta \mathrm{LC})$. A suspension of phage $\alpha$ in Ringer's solution was exposed to the radiation from a Hanovia 'Bactericidal' 15 W. lamp emitting $95 \%$ of its radiation at $2537 \AA$. Samples taken at intervals were plated with non-lysogenic strain Davis and with strain Davis $(\beta \mathrm{LC})$, the same concentration of plating bacteria being used in the two cases.

Fig. 3 shows the numbers of $\alpha$ plaques and $\beta$ plaques produced after a culture of strain Davis $(\beta)$ had been superinfected with phage $\alpha$ at a multiplicity of $9 \cdot 8 /$ chain, together with the numbers of $\beta$ plaques produced by a control culture of strain Davis $(\beta)$ not infected with phage $\alpha$. At the beginning of the experiment, the number of plaques was equal to the calculated number of chains present, therefore, since the free phage had been neutralized by antiserum, it appeared that each chain of strain Davis $(\beta)$ produced a plaque. Three sorts of plaque were seen with the culture infected with phage $\alpha$, namely clear, target and mottled. A number of the mottled plaques were tested and found to give both phage $\alpha$ and phage $\beta$. Each mottled plaque was presumed to have arisen from an infected chain which liberated both sorts of phage, for at 
15 min. the total number of plaques equalled the calculated number of chains, and until $60 \mathrm{~min}$. almost all the plaques were mottled. After this time, when the number of plaques started to increase markedly, the mottled plaques formed a decreasing proportion of the total. The plaque counts have been expressed as multiples of the initial count at $15 \mathrm{~min}$. and have been plotted against time elapsing since the phage and bacteria were mixed; the phage $\alpha$ counts represent the sum of clear and mottled plaques and the phage $\beta$ counts the sum of target and mottled plaques. Infection by phage $\alpha$ evidently induced the prophage in strain Davis $(\beta)$, for there was an increase of phage $\beta$ in the culture infected with phage $\alpha$ which paralleled the increase of phage $\alpha$ and which greatly exceeded its increase in the control culture not infected with phage $\alpha$. A similar result was obtained when the naturally lysogenic strain $W$ was infected with phage $\alpha$.

Ultraviolet-irradiated phage $\alpha$ produced more plaques on lysogenic strain Davis than on non-lysogenic strain Davis (Fig. 4), whereas unirradiated phage $\alpha$ produced only about half as many plaques on lysogenic strain Davis as on non-lysogenic strain Davis.

Jacob \& Wollman (1953) concluded that the reason why the virulent mutant $\lambda \nu$ of the coliphage $\lambda$ can form plaques on strains lysogenic with temperate $\lambda$ is because it can induce the prophage to develop into phage, and in this way convert immune bacteria into sensitive ones in which it can multiply together with the temperate phage. The bacillus phage $\alpha$ resembled coliphage $\lambda \nu$ both in having inducing ability and in being restored by lysogenic strains after inactivation by ultraviolet radiation. It is therefore likely that both phages owe their ability to attack lysogenic hosts to the same cause, and that the arguments applied to coliphage $\lambda \nu$ (Jacob \& Wollman, 1953) apply equally to bacillus phage $\alpha$ : that is, that the virulent phage induces the prophage before it multiplies, and that it is as a consequence of this inducing ability that it is able to multiply in lysogenic strains.

\section{Inducing activity of phage $\gamma$}

Phage $\gamma$ did not form plaques on strains lysogenic with $\beta$ or $\beta \mathrm{LC}$. However, drops of undiluted stocks of phage $\gamma$ spotted on lysogenic strain Davis or lysogenic strain $2160 \mathrm{~S}$ produced patches of complete clearing, and the clearing became less complete with dilution, the effect resembling that obtained with serial dilutions of a colicine. This activity of phage $\gamma$ suspensions was proportional to the concentration of particles forming plaques on non-lysogenic strain Davis, and was destroyed by addition of anti-phage serum. On centrifuging phage preparations at high speed, the activity travelled with the plaqueforming particles (Table 1). Superinfection of strain Davis $(\beta \mathrm{LC})$ with phage $\gamma$ increased the total phage production, the additional phage consisting of both $\gamma$ and $\beta \mathrm{LC}$. In increasing the total phage production, superinfection with phage $\gamma$ differed from superinfection in systems where immunity is evidently complete (Bertani, 1953, 1954; Jacob \& Wollman, 1953), and resembled superinfection with phage $\alpha$. Fig. 5 shows the results of an experiment in which the rate of phage production in a culture of strain Davis $(\beta \mathbf{L C})$ superinfected with 


\section{Table 1. Effect of concentrated phage $\gamma$ on strain Davis $(\beta L C)$}

Ten ml. of a stock of phage $\gamma$ were centrifuged in a Spinco Ultracentrifuge at $21,400 \mathrm{~g}$. for $90 \mathrm{~min}$. The uncentrifuged stock, the top $1 \mathrm{ml}$. of the supernatant fluid, and the deposit resuspended in $1 \mathrm{ml}$. of broth were assayed with strain Davis, and $0.02 \mathrm{ml}$. drops of serial tenfold dilutions were spotted on plates inoculated with strain Davis ( $\beta \mathrm{LC}$ )

\begin{tabular}{|c|c|c|c|}
\hline & Dilution & $\begin{array}{l}\text { Calculated number } \\
\text { of plaque- } \\
\text { producing } \\
\text { particles/drop }\end{array}$ & $\begin{array}{l}\text { Effect on strain } \\
\text { Davis }(\beta \mathrm{LC})\end{array}$ \\
\hline $\begin{array}{l}\text { Uncentrifuged stock (titre on } \\
\text { strain Davis }=1 \times 10^{10} \\
\text { particles } / \mathrm{ml} \text {.) }\end{array}$ & $\begin{array}{l}10^{0} \\
10^{-1} \\
10^{-2} \\
10^{-3} \\
10^{-4}\end{array}$ & $\begin{array}{l}2 \times 10^{8} \\
2 \times 10^{7} \\
2 \times 10^{6} \\
2 \times 10^{5} \\
2 \times 10^{4}\end{array}$ & $\begin{array}{l}\text { Complete clearing } \\
\text { Complete clearing } \\
\text { Partial clearing } \\
\text { Trace } \\
\text { No mark }\end{array}$ \\
\hline $\begin{array}{l}\text { Supernatant fluid (titre on } \\
\text { strain Davis }=5 \times 10^{8} \\
\text { particles } / \mathrm{ml} \text {.) }\end{array}$ & $\begin{array}{l}10^{0} \\
10^{-1} \\
10^{-2}\end{array}$ & $\begin{array}{l}1 \times 10^{7} \\
1 \times 10^{6} \\
1 \times 10^{5}\end{array}$ & $\begin{array}{l}\text { Complete clearing } \\
\text { Trace } \\
\text { No mark }\end{array}$ \\
\hline $\begin{array}{l}\text { Deposit (titre on strain Davis }= \\
5 \times 10^{10} \text { particles } / \mathrm{ml} \text {.) }\end{array}$ & $\begin{array}{l}10^{0} \\
10^{-1} \\
10^{-2} \\
10^{-3} \\
10^{-4}\end{array}$ & $\begin{array}{l}1 \times 10^{9} \\
1 \times 10^{8} \\
1 \times 10^{7} \\
1 \times 10^{6} \\
1 \times 10^{5}\end{array}$ & $\begin{array}{l}\text { Complete clearing } \\
\text { Complete clearing } \\
\text { Complete clearing } \\
\text { Trace } \\
\text { No mark }\end{array}$ \\
\hline
\end{tabular}

phage $\gamma$ at a multiplicity of $0 \cdot 1 /$ chain was compared with that in a control culture without phage $\gamma$. Samples were plated with streptomycin-resistant strain Davis on streptomycin agar to eliminate the background of plaques produced by chains of lysogenic bacteria. It will be seen that phage $\gamma$ is able to some extent to induce the development of prophage $\beta \mathbf{L C}$ to phage, for more phage $\beta \mathbf{L C}$ was produced in the culture superinfected with phage $\gamma$, and that because of this inducing ability it is itself able to multiply to some extent, for the $\gamma$ plaques increased in parallel with the extra $\beta$ LC plaques.

The growth curve of phage $\gamma$ in strain Davis $(\beta \mathrm{LC})$ differed considerably from that of the phages in systems where plaques were formed, e.g. any of the phages in non-lysogenic strain Davis or phage $\alpha$ in lysogenic strain Davis. In one-step growth experiments with any of the phages in non-lysogenic strain Davis or phage $\alpha$ in lysogenic strain Davis, the minimum latent period was about 65 min.* Three striking differences could be observed between the growth curve of phage $\gamma$ in strain Davis $(\beta \mathrm{LC})$ and the growth curves of the phages in systems where plaques were formed. (1) Only a small proportion $(1 / 350)$ of the bacteria which had adsorbed phage $\gamma$ gave rise to plaques on streptomycin agar at $60 \mathrm{~min}$. suggesting that only about this proportion then

* The technique of the one-step growth experiment does not give true one-step growth curves when the host grows in chains, for phage particles released in the first burst re-adsorb to other bacteria in the chain from which they came, and go through a further cycle of multiplication, and this second cycle of multiplication cannot be avoided by dilution as in the ordinary one-step growth curve (McCloy, to be published.) However, in one-step growth experiments with any of the phages in non-lysogenic strain Davis or phage $\alpha$ in lysogenic strain Davis, a marked decline in the rate of increase of plaque-forming particles occurred at about $100 \mathrm{~min}$., probably indicating that the majority of the bacteria infected in the first cycle had released their phage by this time; and from the number of plaques at, or soon after 100 min., the mean burst size could be estimated to be of the order of 100 . 
contained mature phage (see Methods). With systems where plaques were formed, half or more of the infected bacteria would have produced plaques on streptomycin agar by this time. This suggests that not more than $1 / 175$ of the bacteria which had absorbed phage $\gamma$ contained mature phage by this time. (2) Even as late as $\mathbf{2 5 5} \mathrm{min}$. the increase of phage over that initially absorbed

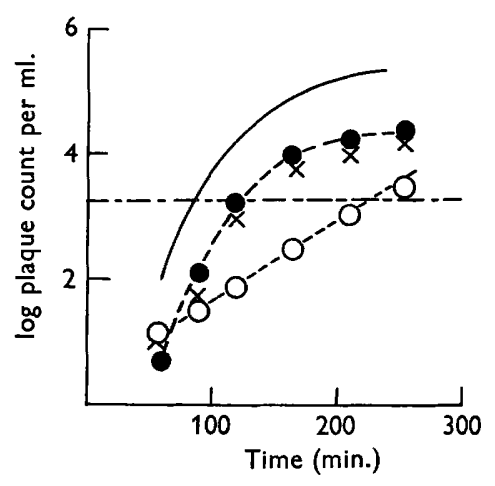

a

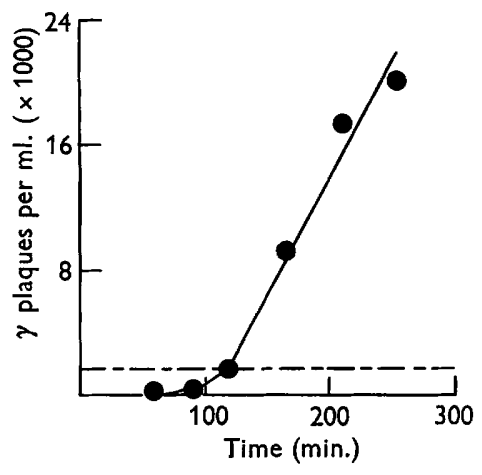

b

Fig. $5 a, b$. Superinfection of strain Davis $(\beta \mathbf{L C})$ with phage $\gamma$. A broth culture of strain Davis $(\beta \mathrm{LC})$ in the logarithmic phase of growth was centrifuged, the deposit was resuspended in a smaller volume of fresh broth to a concentration of $1.25 \times 10^{8}$ chains $/ \mathrm{ml}$. as estimated by colony count, and divided into two $0.9 \mathrm{ml}$. volumes. $0.1 \mathrm{ml}$. of phage $\gamma$ was added to one giving a concentration of $1.75 \times 10^{7} / \mathrm{ml}$., and $0 \cdot 1 \mathrm{ml}$. of broth was added to the other. After $10 \mathrm{~min}$. for adsorption, both suspensions were treated with antiphage serum to inactivate the free phage present. $99 \%$ of the phage $\gamma$ was absorbed, as estimated by centrifuging a dilution made without anti-phage serum and assaying the supernatant. Suitable dilutions of each suspension in broth were incubated at $37^{\circ}$, and samples taken at intervals were plated with streptomycin-resistant strain Davis on streptomycin agar.

(a) shows log concentration of $\gamma$ plaques $(\bullet)$ and $\beta$ LC plaques $(x)$ produced by the superinfected culture, together with $\beta$ LC plaques produced by the control culture $(O)$ plotted against time after addition of phage $\gamma$.

(b) shows the arithmetic concentration of $\gamma$ plaques $(\bullet)$ plotted against time after addition of phage $\gamma$.

Continuous lines. Theoretical curves for the increase of phage $\gamma$ in cultures of strain Davis $(\beta L C)$ derived on the assumptions (1) that the phage in 1/175 of the infected bacteria reaches maturity every $5 \mathrm{~min}$. from $60 \mathrm{~min}$. onwards, and (2) that, after maturation, infective centres increase at the same rate as in systems where plaques are formed. The curve in $(a)$ has been displaced upwards by one log unit for clarity.

Broken lines. Concentration of phage $\gamma$ adsorbed to the bacteria.

was only 12-fold, whereas with systems where plaques were formed it was about 100-fold at $120 \mathrm{~min}$. (3) With systems where plaques were formed, a decline in the rate of increase of plaque-forming particles occurred at 100$120 \mathrm{~min}$; in this case, the number of plaques was still increasing linearly at $210 \mathrm{~min}$. It seems very unlikely that the phage $\gamma$ present at $210 \mathrm{~min}$. was derived solely from those bacteria which contained mature phage $\gamma$ at $60 \mathrm{~min}$.; more probably intracellular maturation of phage $\gamma$ in strain Davis $(\beta \mathrm{LC})$ was not synchronized to the same extent as that of phage in systems where plaques were formed.

It is clearly impossible from a curve such as that of Fig. 5 to determine what 
proportion of the infected bacteria did eventually burst, but to get some idea of what proportion of bacteria of strain Davis $(\beta \mathrm{LC})$ which had absorbed phage $\gamma$ did ultimately release phage $\gamma$, suspensions of bacteria of strain Davis $(\beta \mathrm{LC})$ were infected with phage $\gamma$ at low multiplicity, and, after dilution through anti-phage serum, plated with non-lysogenic strain Davis on agar without streptomycin. After incubation, a number of normal-sized clear plaques and a few normal-sized mottled plaques could be distinguished and counted fairly satisfactorily, despite the presence of a background of small plaques arising from micro-colonies of the lysogenic strain. Control suspensions of strain Davis $(\beta \mathrm{LC})$ without phage $\gamma$, plated at the same time, produced neither clear nor mottled plaques. In one experiment, $1.4 \times 10^{4} \gamma$ particles were absorbed and $2.8 \times 10^{3}$ normal-sized clear plaques resulted; in another experiment 9.8 $\times 10^{4}$ $\gamma$ particles were absorbed and $1 \cdot 6 \times 10^{4}$ normal-sized clear plaques resulted. Thus, in the first experiment $21 \%$, and in the second experiment $16 \%$ of the $\gamma$ particles which adsorbed to bacteria of strain Davis $(\beta \mathbf{L C})$ multiplied soon enough to give normal-sized plaques when plated with non-lysogenic strain Davis.

The small increase in phage $\gamma$ over the input in the experiment of Fig. 5 might be the result of the bursting of all of this fraction during the period of the experiment ( $255 \mathrm{~min}$.), with a smaller burst size and a latent period which was more variable, but only slightly longer than the usual. Or it might be the result of a situation where the burst size was of the usual order, and where only a proportion of the bursts occurred within the period of observation, the period between infection and bursting varying between the normal and at least $255 \mathrm{~min}$., at which time the plaque count was still rising. From 120 to $210 \mathrm{~min}$. the plaque count increased approximately linearly with time (the curvature of the line in Fig. $5 a$ results from the use of semi-log co-ordinates as may be seen by comparison with Fig. $5 b$ which shows the same data plotted arithmetically). Thus phage was being released at a constant rate instead of all at about the same time as in a normal one-step growth curve. It is possible that superinfecting phage $\gamma$ becomes mature in a constant proportion of the infected bacteria/unit time, and that once maturation has started the cell bursts after the same interval and with the same burst size as in systems where plaques are formed; the curve of increase of plaques predicted by a model of this sort, assuming that the phage in $1 / 175$ of the infected bacteria reaches maturity every 5 min. from 60 min. onwards, is shown as the continuous lines in Fig. 5 a and $5 b$, and is very similar to the curve actually observed.

Since the latent period of phage $\alpha$ is the same in strain Davis $(\beta \mathbf{L C})$ as in nonlysogenic strain Davis (Fig. 2), one may assume that the induction process initiated by phage $\alpha$ occupies a negligible time, and that its latent period of actual multiplication is no longer in strain Davis $(\beta \mathrm{LC})$ than in non-lysogenic strain Davis. It is reasonable to suppose that the same is true of phage $\gamma$, and hence that the slow rate of intracellular maturation of phage $\gamma$ is due to delay in causing induction after entry into the bacterium. Perhaps not all the particles which eventually induce start to induce at once. Particles of phage $\gamma$ when they invade bacteria carrying prophage may remain viable, like other 
superinfecting phages, not inert as in systems where immunity is complete (Bertani, 1953, 1954; Jacob \& Wollman, 1953) but with a certain probability/ unit time of inducing the prophage to develop into phage and subsequently multiplying. The slow rate of growth of phage $\gamma$ in strain Davis ( $\beta \mathrm{LC}$ ) is probably responsible for its failure to form plaques on this strain. This hindrance might be overcome if a large number of phage particles were inoculated at one point. This was done by plating bacteria of non-lysogenic strain Davis infected with phage $\gamma$, each of which would liberate $c .100$ phage particles at one point, instead of free phage $\gamma$, and visible though very small plaques were in fact produced (Pl. 1, fig. 6). Their numbers in two experiments were 25 and $50 \%$ of the numbers of plaques produced on non-lysogenic strain Davis.

\section{Inducing activity of phage $\beta$}

Although phage $\beta$ did not form plaques on bacterial strains lysogenic with $\beta$ itself or with $\beta \mathrm{LC}$, drops of concentrated phage $\beta$ spotted on lysogenic strains Davis or $2160 \mathrm{~S}$ produced patches of clearing resembling those produced by phage $\gamma$, the activity of suspensions being proportional to their concentration of plaque-forming particles on non-lysogenic strain Davis. As can be seen in Table 2 , the activity of phage $\beta$ per plaque-forming particle was about 10 times less than that of phage $\gamma$. The clearing was due to phage $\beta$ itself and not to the presence of phage $\gamma$ contaminating the suspensions.

\section{Table 2. Effect of concentrated phage $\beta$ on strain Davis $(\beta L C)$}

Two $10 \mathrm{ml}$. volumes of phage $\beta$ were centrifuged in a Spinco Ultracentrifuge at $21,400 \mathrm{~g}$. for $90 \mathrm{~min}$. The uncentrifuged stock, the pooled supernatant fluids, and the pooled deposits resuspended in $1 \mathrm{ml}$. of broth, were assayed with strain Davis, and $0.02 \mathrm{ml}$. drops of serial 10-fold dilutions were spotted on plates spread with strain Davis $(\beta \mathbf{L C})$.

\begin{tabular}{|c|c|c|c|}
\hline & Dilution & $\begin{array}{l}\text { Calculated number of } \\
\text { plaque-producing } \\
\text { particles/drop }\end{array}$ & $\begin{array}{c}\text { Effect on strain } \\
\text { Davis }(\beta L C)\end{array}$ \\
\hline $\begin{array}{l}\text { Uncentrifuged stock (titre } \\
\text { on strain Davis }=1 \cdot 1 \times 10^{10} \\
\text { particles } / \mathrm{ml} \text {.) }\end{array}$ & $\begin{array}{l}10^{0} \\
10^{-1} \\
10^{-2} \\
10^{-3}\end{array}$ & $\begin{array}{l}2 \times 10^{8} \\
2 \times 10^{7} \\
2 \times 10^{6} \\
2 \times 10^{5}\end{array}$ & $\begin{array}{l}\text { Partial clearing } \\
\text { Partial clearing } \\
\text { Trace } \\
\text { No mark }\end{array}$ \\
\hline $\begin{array}{l}\text { Supernatant fluid (titre on } \\
\text { strain Davis }=4 \cdot 3 \times 10^{8} \\
\text { particles/ml.) }\end{array}$ & $\begin{array}{l}10^{0} \\
10^{-1}\end{array}$ & $\begin{array}{l}8.6 \times 10^{6} \\
8.6 \times 10^{5}\end{array}$ & $\begin{array}{l}\text { Trace } \\
\text { No mark }\end{array}$ \\
\hline $\begin{array}{l}\text { Deposit (titre on strain } \\
\text { Davis }=1 \cdot 8 \times 10^{11} \text { particles/ } \\
\text { ml.) }\end{array}$ & $\begin{array}{l}10^{0} \\
10^{-1} \\
10^{-2} \\
10^{-3} \\
10^{-4}\end{array}$ & $\begin{array}{l}3 \cdot 6 \times 10^{9} \\
3 \cdot 6 \times 10^{8} \\
3 \cdot 6 \times 10^{7} \\
3 \cdot 6 \times 10^{6} \\
3 \cdot 6 \times 10^{5}\end{array}$ & $\begin{array}{l}\text { Complete clearing* } \\
\text { Complete clearing* } \\
\text { Partial clearing } \\
\text { Trace } \\
\text { No mark }\end{array}$ \\
\hline
\end{tabular}

* Or with a few colonies on a clear base.

The ability of phage $\gamma$ to induce and to multiply in strain Davis $(\beta \mathrm{LC})$ suggested that the clearing produced by phage $\beta$ was due to the same cause. This hypothesis was supported by the shape of the curve of phage production in cultures of strain Davis $(\beta)$ (Fig. 1a). In such cultures, after a period during 
which the titre of phage $\beta$ decreased, evidently as a result of re-adsorption to the bacteria, there was a second rise in the concentration of phage, presumably due to induction following superinfection. Furthermore, it was shown that superinfection of strain Davis $(\beta)$ by added phage $\beta$ increased the production of

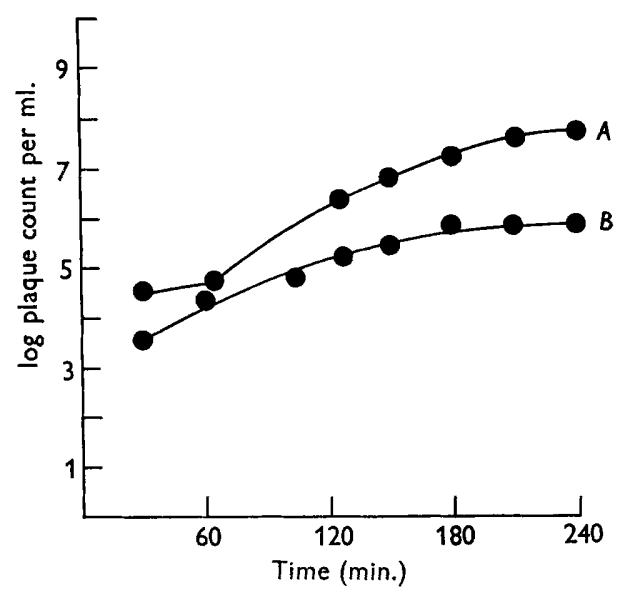

Fig. 6

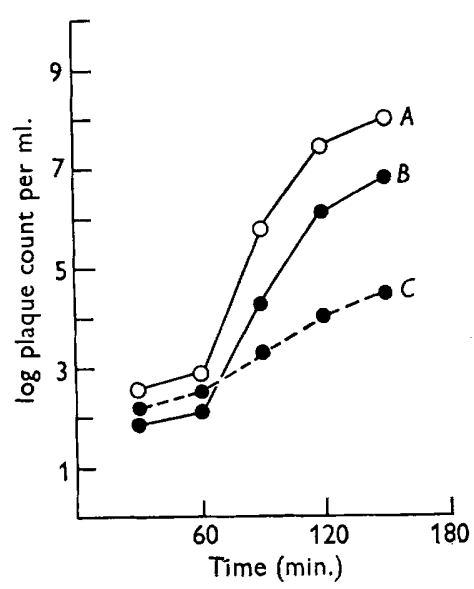

Fig. 7

Fig. 6. Superinfection of strain Davis $(\beta)$ with phage $\beta$. A broth culture of strain Davis $(\beta)$ towards the end of the logarithmic phase of growth was centrifuged, and the deposit was resuspended in fresh broth to a turbidity approximately corresponding to that of $5 \times 10^{8}$ Escherichia coli $/ \mathrm{ml}$. One $\mathrm{ml}$. was placed in each of two tubes, and $0.05 \mathrm{ml}$. of a stock of phage $\beta$ was added to one giving a concentration of $8.5 \times 10^{\%} / \mathrm{ml}$. After $8 \mathrm{~min}$. for adsorption of the added phage, both suspensions were treated with anti-phage serum to inactivate the free phage present. 1/200 dilutions of the suspensions in $50 \mathrm{ml}$. volumes of broth were incubated at $37^{\circ}$, and samples taken at intervals were diluted and plated with streptomycin-resistant strain Davis on streptomycin agar.

The log concentrations of plaques (all $\beta$ ) produced by the superinfected culture (curve $A$ ), and by the control culture (curve $B$ ) are plotted against time after addition of phage $\beta$.

Fig. 7. Superinfection of strain Davis $(\beta \mathrm{LC})$ with phage $\beta$. A broth culture of strain Davis $(\beta \mathrm{LC})$ in the logarithmic phase of growth was centrifuged, and the deposit was resuspended in fresh broth to a turbidity approximately corresponding to that of $10^{9}$ Escherichia coli $/ \mathrm{ml}$. Equal volumes were placed in each of two tubes, and phage $\beta$ was added to one to a concentration of $8 \times 10^{9} / \mathrm{ml}$. After $10 \mathrm{~min}$. for adsorption of the added phage, both suspensions were treated with anti-phage serum to inactivate the free phage present. Suitable dilutions in broth were incubated at $37^{\circ}$, and samples taken at intervals were plated with streptomycin-resistant strain Davis on streptomycin agar. The log concentrations of $\beta$ plaques (curve $A$ ), and $\beta$ LC plaques (curve $B$ ) produced by the superinfected culture, together with $\beta$ LC plaques produced by the control culture (curve $C$ ) are plotted against time after addition of phage $\beta$. (A number of minute plaques were observed with both eultures in this experiment, and in other experiments where lysogenic strains were plated with streptomycin-resistant strain Davis on streptomycin agar. These have not been included in the counts given. Their significance is uncertain, but there is some evidence which suggests that they may be due to the occurrence of reversible adsorption of phage to lysogenic strains.)

phage $\beta$ (Fig. 6). This effect might conceivably be due to the added phage $\beta$ infecting bacteria which had spontaneously become non-lysogenic (Clarke, 1952; Bertani, 1958), but since the spontaneous rate of production of phage $\beta$ by strain Davis $(\beta)$ and the rate of adsorption of phage $\beta$ to strain Davis $(\beta)$ 
are both high, it is unlikely that non-lysogenic bacteria, if they arose, would remain uninfected by phage $\beta$ naturally present in the culture. It follows that the increase in phage $\beta$ was probably a sequel of superinfection of lysogenic bacteria and induction. This was proved by superinfecting strain Davis $(\beta \mathrm{LC})$ with phage $\beta$, for it was then found that both phages contributed to the excess of phage found by comparison with a control culture of strain Davis $(\beta \mathrm{LC})$ without phage $\beta$ (Fig. 7).

\section{Phage $\beta$}

\section{Appearance of the phages in cultures of lysogenic strains}

Phage $\beta$ was produced regularly during growth of the naturally lysogenic strain $W$ and of artificially lysogenic strains.

\section{Phage $\gamma$}

Phage $\gamma$ could be detected during a certain period of the growth of broth cultures of bacterial strains lysogenic with phage $\beta$ started from inocula in which the free phage had been inactivated, either by using a heated spore suspension, or, in the case of the asporogenous strains, by treating them with anti-phage serum. When samples from such cultures were plated with nonlysogenic streptomycin-resistant indicator strains, Davis or $2160 \mathrm{~S}$, on streptomycin agar, there were some clear plaques as well as the usual targetlike $\beta$ plaques. A number of these clear plaques were tested; all gave phage which formed clear plaques on non-lysogenic strain Davis, and did not form plaques on either lysogenic strain Davis or strain $\mathbf{W}$, and which was thus by definition phage $\gamma$. Clear plaques caused by phage $\gamma$ were only rarely seen with cultures started from inocula in which the free phage $\beta$ had not been inactivated, and which therefore contained more phage $\beta$ in the initial stages of their growth.

When growing cultures of strain W started from inocula of heated spores were examined, it was seen that with the first samples to produce any plaques, the number of $\gamma$ (clear) plaques was about a tenth to a twentieth the number of $\beta$ plaques. As the cultures grew and the plaques became more numerous, the $\gamma$ plaques formed a progressively diminishing proportion of the total, and soon after the cultures had gone out of the logarithmic phase of growth clear plaques could no longer be detected; there is no selective system for phage $\gamma$ in the presence of a great excess of phage $\beta$. In experiments where the free phage in the inoculum was not inactivated, clear plaques were only occasionally seen. It thus appeared that phage $\gamma$ could arise in lysogenic cultures, and that if there was no free phage present initially, it formed a considerable, but progressively diminishing, proportion of the total phage during the earlier phase of growth of the culture.

The appearance of phage $\gamma$ in these cultures of strain $W$ was presumably due to a mutation of phage $\beta$. If only phage $\beta$ in the form of lytic phage could mutate, then it would be expected that the number of $\gamma$ plaques would form a constant proportion of the number of $\beta$ plaques instead of a diminishing proportion as was found. Ultraviolet irradiation could induce the naturally 
lysogenic strain $\mathrm{W}$ to some extent; when cultures at a stage in their growth when phage $\gamma$ was detectable were irradiated, an increased production of phage $\beta$ was obtained, sometimes up to 40 -fold of that in a control unirradiated culture, but this rise in phage $\beta$ was never accompanied by a rise in phage $\gamma$. The findings are most easily explained by assuming that mutation from $\beta$ to $\gamma$ occurs in the prophage, and that the $\gamma$ thus produced multiplies as lytic phage, which is likely since phage $\gamma$ cannot lysogenize. There is no selective system for phage $\gamma$ in the presence of phage $\beta$, but a streptomycin-resistant indicator on streptomycin agar is a selective indicator for phage $\gamma$ in the presence of prophage $\beta$. Assuming that mutation to $\gamma$ occurred in both prophage $\beta$ and phage $\beta$ at a low rate, the mutation could be detected when there was enough prophage $\beta$ to give some $\gamma$ mutants but not enough phage $\beta$ to mask them. The conditions in cultures starting from phage-free inocula would thus favour the detection of such a mutation.

This hypothesis was supported by the results of a single-burst experiment in which broth inoculated with heated spores of strain $W$ was distributed in small volumes which, after a period of incubation, were plated with streptomycinresistant indicator strain $2160 \mathrm{~S}$ on streptomycin agar. If phage $\gamma$ arose by mutation of prophage $\beta$ and subsequently multiplied, the bacterium in which this occurred might yield only phage $\gamma$. If it arose only by mutation of lytic phage $\beta$, the bacterium in which the mutation occurred would be expected to yield both phages. In this experiment, 204 samples were plated and of these 109 gave no plaques, 91 gave only $\beta$ plaques, 3 gave only $\gamma$ plaques, and 1 gave both $\gamma$ and $\beta$ plaques. The proportion giving both $\gamma$ and $\beta$ plaques was no higher than that to be expected if the two events were independent of each other, which suggests that this tube contained the yield of more than one bacterium.

\section{Phage $\alpha$}

In cultures of the naturally lysogenic strain W. Broth cultures of strain W incubated for prolonged periods regularly came to contain large amounts of phage $\alpha$. Loopfuls plated on nutrient agar gave rise to bacterial growth with eroded patches from which phage $\alpha$ could be isolated, and phage $\alpha$ was also found in filtrates of the broth cultures, reaching concentrations of about $10^{9}$ particles/ml. The time of incubation before a broth culture gave eroded growth on plating varied between 2 and 25 days, and was perhaps shorter when the volume of the culture was large. The method of plating a loopful does not show when phage $\alpha$ first makes its appearance in the culture but reveals it only when it has multiplied to the concentration necessary for particles to be present in the volume held by the loop, that is, to above about $10^{3}$ particles $/ \mathrm{ml}$. Tests for the first appearance of phage $\alpha$ consisting of plating the whole of cultures of small volume could not satisfactorily be made because : $(a)$ the appearance of a few plaques in a lawn of a strain $W$ does not necessarily mean that the particles originating the plaques were present in the inoculum (see below); (b) phage $\alpha$ sensitive strains other than strain $W$ were also sensitive to a bacteriocine which strain $W$ produces, and thus a strain lysogenic with $\beta \mathrm{LC}$ could not be used as an indicator for phage $\alpha$ with undiluted fully grown cultures of strain $W$. 
Addition of glucose, mercaptoacetate, calcium, citrate or oxalate to Lemco broth did not seem to alter the time of appearance of phage $\alpha$; but addition of manganese hastened its appearance (Table 3). Addition of manganese to the broth gave an increased final crop of bacteria as judged by turbidity measurements, but a still higher concentration of bacteria obtained by aeration did not

Table 3. Effect of volume and composition of medium on time of appearance of phage $\alpha$ in cultures of strain $W$

Number of cultures first showing phage $\alpha$ on

Total (days of incubation at $37^{\circ}$ ):

\begin{tabular}{|c|c|c|c|c|c|c|c|c|c|c|c|c|c|c|c|c|c|c|c|c|}
\hline Day $\ldots$ & 1 & 2 & $\mathbf{3}$ & 4 & 5 & 6 & 78 & 8 & 9 & 10 & 11 & 12 & 13 & 141 & 151 & 161 & 1718 & $\begin{array}{ll}819 \\
\end{array}$ & 20 & tested \\
\hline $\begin{array}{l}100 \mathrm{ml} \text {. Lemco } \\
\text { broth }\end{array}$ & . & 3 & 3 & 6 & 61 & 11 & 5 & . & 1 & 1 & . & . & - & . & & . & 1 & - & 2 & 39 \\
\hline $\begin{array}{c}100 \mathrm{ml} \text {. Lemco } \\
\text { broth shaken }\end{array}$ & . & . & . & 2 & . & 1 & 1 & 1 & - & 2 & 1 & - & - & - & . & - & • & . & . & 8 \\
\hline $\begin{array}{r}100 \mathrm{ml} . \text { Lemco } \\
\text { broth }+\mathrm{MnCl}_{2}\end{array}$ & 1 & 9 & 5 & 1 & . & . & . & - & . & . & - & - & - & - & - & . & . & • & . & 16 \\
\hline $\begin{array}{l}5 \mathrm{ml} . \text { Lemco } \\
\text { broth }\end{array}$ & . & . & 1 & 1 & . & 1 & $3:$ & 3 & 1 & . & 3 & - & - & & 4. & . & . & . & 1 & 18 \\
\hline $\begin{array}{l}5 \mathrm{ml} \text {. Lemco } \\
\text { broth }+\mathrm{MnCl}_{2}\end{array}$ & 1 & 5 & 4 & . & . & 2 & - & . & - & . & . & . & - & & & . & • & - & . & 12 \\
\hline
\end{tabular}

hasten the appearance of detectable amounts of phage $\alpha$. Nor did concentration of the bacteria by centrifuging $24 \mathrm{hr}$. cultures and incubating the deposits resuspended in smaller volumes (1/5 to $1 / 2)$ of the original medium. The concentrations of phage $\beta$ in cultures with and without added manganese, at times before phage $\alpha$ was present in detectable amount, were not appreciably different. The effect of added manganese might have been on the rate of multiplication of phage $\alpha$ to a detectable concentration; but in experiments to test this addition of manganese did not noticeably increase the rate of multiplication of phage $\alpha$ added to log phase cultures or $24 \mathrm{hr}$. cultures of strain W.

Spontaneous plaques of phage $\alpha$ were sometimes seen when inocula of strain $W$ apparently containing no phage $\alpha$ were plated. These plaques were more numerous on nutrient agar containing liver extract than on unsupplemented nutrient agar, and were more numerous after incubation for $48 \mathrm{hr}$. than for $24 \mathrm{hr}$. Since bacterial growth was more profuse on liver agar than on ordinary nutrient agar, the more numerous plaques on liver agar did not necessarily mean a greater rate of production of phage $\alpha$ on this medium.

In cultures of the artificially lysogenic derivatives of sensitive bacterial strains. Broth cultures of the lysogenic derivatives of sensitive bacterial strains obtained by exposure to phage $\beta$ also came to contain high concentrations of phage $\alpha$ when incubated for long enough. The time at which phage $\alpha$ was detectable by plating a loopful of the culture was earlier with the rapidly absorbing atypical Bacillus anthracis strains Davis $(\beta)$, Lindsay $(\beta)$ and smooth lysogenic derivatives of strain 2160 than with typical $B$. anthracis strains or $\boldsymbol{B}$. cereus strains, phage $\alpha$ being not uncommonly present in large amount in 
cultures of the atypical $B$. anthracis strains after only $24 \mathrm{hr}$. of incubation. Spontaneous plaques caused by phage $\alpha$ were also seen in cultures on agar; they were especially frequent with the atypical $B$. anthracis strains.

\section{Phage $\beta$ to phage $\gamma$}

\section{Interconversions}

As described above, phage $\gamma$ could be found in cultures of bacterial strains lysogenic with phage $\beta$, apparently as a result of mutation in prophage $\beta$. Spontaneous mutation of lytic phage $\beta$ to $\gamma$ could not be demonstrated; while, as argued above, plating on streptomycin agar is a selective system for phage $\gamma$ in the presence of an excess of prophage $\beta$, there is no selective system for phage $\gamma$ in the presence of a great excess of phage $\beta$, so small amounts of phage $\gamma$ could not be detected in stocks of phage $\beta$. A phage with the properties of phage $\gamma$ arose from phage $\beta$ when it was irradiated with u.v. radiation and plated with bacteria which had also been irradiated (Weigle, 1953). When phage $\beta$ irradiated with a dose of ultraviolet radiation which gave a survival of $10^{-6}$ on plating with unirradiated strain Davis was plated with irradiated bacteria, the survival was $10^{-5}$, and some of the plaques which were produced were clear. The clear plaques yielded phage which formed clear plaques on nonlysogenic strain Davis and no plaques on lysogenic strain Davis, and thus had the properties of phage $\gamma$.

\section{Phage $\beta$ to phage $\alpha$}

Phage $\alpha$ could be found in cultures of bacterial strains lysogenic with phage $\beta$. It could also be found in stocks of phage $\beta$ grown on sensitive strains by plating with the selective indicator strains Davis $(\beta \mathrm{LC})$ and $2160(\beta) \mathrm{S}$, though here it was only detected in small amounts, e.g. a proportion of about 1 in $2 \times 10^{6}$. There was probably more phage $\alpha$ present than was detected, for tests on the sensitivity of the assay system made by making parallel titrations of a stock of phage $\alpha$ diluted in broth and diluted in a stock of phage $\beta$ of titre $5 \times 10^{8}$ particles $/ \mathrm{ml}$. showed that the presence of this concentration of phage $\beta$ lowered the efficiency of plating of phage $\alpha$ to about $1 / 6$ to $1 / 10$, and caused the plaques to be small and indistinct. In contrast to the situation in cultures of lysogenic strains, phage $\alpha$ was evidently at no selective advantage over phage $\beta$ in cultures of non-lysogenic bacterial strains. When a mixture of phage $\alpha$ and phage $\beta$ was serially passaged five times with non-lysogenic strain Davis, with mass transfers of phage at each step, the relative proportions of the two phages remained the same throughout.

\section{Phage $\alpha$ to phage $\beta$}

Phage $\beta$ was obtained from phage $\alpha$ only in one experimental system. Bacilli of sensitive strains could sporulate despite infection with phages $\alpha$ or $\gamma$ (McCloy, 1953; and to be published). A large proportion, about $0 \cdot 5-0 \cdot 1$, of the spores of Bacillus anthracis strain 1444 formed in the presence of phage $\alpha$ were found to be lysogenic with phage $\beta$. Phage $\beta$ could evidently arise during growth of phage $\alpha$ in strain 1444, for spores lysogenic with phage $\beta$ were found 
when single plaques of phage $\alpha$ on strain 1444 were picked and replated with strain 1444.

No phage $\beta$ was found when phage $\alpha$ was grown on strain Davis. The secondary bacterial growth in about 20 cultures of strain Davis which had been lysed by phage $\alpha$ was examined, but no bacteria lysogenic with phage $\beta$ were found. In one experiment, $100 \mathrm{ml}$. of broth were inoculated with strain Davis and phage $\alpha$, and incubated until lysis had been followed by secondary growth. The culture was then centrifuged, and half the supernatant fluid +an equal volume of fresh broth was inoculated with a large amount of strain Davis, and again incubated until the culture had cleared and secondary growth had appeared. Fourteen such subcultures were made and at each step the deposit was examined for bacteria lysogenic with phage $\beta$, but none were found.

Since strains lysogenic with phage $\beta$ are still susceptible to phage $\alpha$, the difference between the results in the two systems tested need not have been due to a difference in the frequency with which phage $\beta$ arose from phage $\alpha$ in the two hosts; it might merely have been due to the difficulty of detecting phage $\beta$ in the presence of phage $\alpha$. Bacilli of Bacillus anthracis strain 1444 absorb the phage slowly, and so single infections might be less rare with this strain than with strain Davis which absorbs rapidly. Furthermore, a bacillus just about to sporulate might absorb only one phage particle before its sporulation was completed, and spores (which do not absorb) containing phage $\beta$ would be completely protected from particles of phage $\alpha$ in their environment.

\section{Phage $\alpha$ to phage $\gamma$}

Since there was no selective indicator for phage $\gamma$ in the presence of phage $\alpha$, there were no means of detecting small amounts of phage $\gamma$ in the presence of large amounts of phage $\alpha$. There was not enough phage $\gamma$ in stocks of phage $\alpha$ to be detected without a selective indicator; some hundreds of plaques produced when preparations of phage $\alpha$ were plated with non-lysogenic bacterial strains were examined, but none were found to contain phage $\gamma$.

\section{Phage $\gamma$ to phage $\beta$}

When the secondary growth in each of about 30 broth cultures of strain Davis which had been cleared by phage $\gamma$ was examined, bacteria lysogenic with phage $\beta$ were found in two of them. Thus phage $\beta$ could arise from phage $\gamma$ though its appearance was only rarely observed. When the spores of Bacillus anthracis strain $\mathbf{1 4 4 4}$ formed in the presence of phage $\gamma$ were examined, no spores lysogenic with phage $\beta$ were found, although an extensive search was made for them, whence it may perhaps be inferred that phage $\beta$ arose from phage $\gamma$ much more rarely than from phage $\alpha$.

\section{Phage $\gamma$ to phage $\alpha$}

Stocks of phage $\gamma$ could be tested for the presence of phage $\alpha$ by plating with indicator strains lysogenic with phages $\beta$ or $\beta \mathrm{LC}$, but only a limited concentration of phage $\gamma$ could be plated because of the clearing which phage $\gamma$ itself 
produced in high concentration. Phage $\alpha$ was found in many stocks of phage $\gamma$ in a proportion of about 1 in $5 \times 10^{6}$.

Phage $\alpha$ was not observed to give rise to phage $\gamma$, but there was no selective indicator available to detect small amounts of phage $\gamma$ in the presence of large amounts of phage $\alpha$. With this one exception, each phage variant was observed to give rise to each of the other two without large amounts of the third being present. It may be inferred from this that each form of the phage could be obtained from either of the other two without passing through the third, which suggests that the determinants of the three forms were probably allelic.

\section{DISCUSSION}

Bacillus cereus strain $\mathbf{W}$ carries a temperate phage $\mathrm{W} \beta$ which, apparently as a result of a single mutation, gives rise to a variant $W \alpha$ which can form plaques on strain $W$ itself and on other bacterial strains lysogenic with the temperate phage. It can do this probably because it is able to induce the prophage to develop into phage, and thus overcome the immunity which is conferred on the bacteria by the prophage. Bacterial strains which give eroded growth are not uncommonly seen, and the isolation of such strains has been reported by several authors including Twort (1915), Scholtens (1950) and Fisk (1942). It is probable that these are lysogenic strains analogous to strain $W$ in that the temperate phage they carry frequently mutates to an inducing virulent form. Evidently the lysogenic culture provides an enrichment medium for mutants of this sort. This mechanism might therefore explain the early reports of spontaneous generation of phage during serial passage of bacteria with supernatant fluids or filtrates from cultures of the same strain, and may in some cases have been misinterpreted as the adaptation by serial passage of an added phage to attack a given strain of bacteria.

It is conceivable that the phage carried by any lysogenic strain could mutate to an inducing virulent variant, the reason why such variants have been found with only a few phages being that the others are many mutational steps from the inducing virulent form. The genetics of phage $\lambda$ have been fully analysed (Jacob \& Wollman, 1954; Kaiser, 1955) and wild-type $\lambda$ has been found to differ by several genes from the inducing virulent $\lambda v$.

The genetic determination of characters in phage $\mathbf{W}$ evidently differs somewhat from that in phage $\lambda$; in phage $\lambda$ there are three alleles at one locus, only one of which, $v 1$, determines the ability to induce. Phages with either of the other two, $c$ (determining clear plaques and inability to lysogenise) or $c^{+}$ (determining turbid plaques and ability to lysogenize), do not induce. The degree of inducing ability of phage with the gene $v_{1}$ (i.e. the probability per phage particle of inducing) is determined by genes at three other loci. In phage $\mathbf{W}$, there are evidently three allelic genes $\beta, \gamma$ and $\alpha$, at one locus which appear superficially to correspond to $c^{+}, c$ and $v_{1}$ in phage $\lambda$, from which however they differ in that each is associated with some inducing ability, the extent of this being determined by which is present. Even the $\beta$ allele which lysogenizes is associated with some degree of inducing activity. The inducing 
activity of phage $\beta$ in lysogenic cultures manifests itself by a form of autolysis when the bacterial strain absorbs the phage readily and is slowly growing. For example, the growth of strain Davis $(\beta)$ is thinner than that of non-lysogenic strain Davis, and the centres of individual colonies are hollowed out. Although the effect of lysogenicity on the colonial morphology of the naturally lysogenic Bacillus cereus strain $\mathbf{W}$ could not be directly determined because no nonlysogenic derivatives of strain $W$ were obtained, it seemed unlikely that carriage of phage $\beta$ was affecting the colonial appearance of this strain since it resembled that of other strains of $\boldsymbol{B}$. cereus. Lawns of strain $W$ were occasionally seen which were finely pitted, and these pits may possibly have been associated with the inducing effect of phage $\beta$, for they were evidently not due either to spontaneously-appearing phage $\alpha$ or to sporulation.

With a few exceptions (Burnet \& Lush, 1936; Bradley \& Boyd, 1952; Boyd, 1954), the bacteria of lysogenic strains absorb the phage that they produce. However, in all systems reported so far, the lysogenic strain is immune to the phage that it produces (Bertani, 1958) although in most cases the only test for immunity that has been used is the ability of the phage to produce clearing of the lysogenic strain. In some cases where more detailed examination has been made (Bertani, 1954; Jacob \& Wollman, 1953), it has been shown that superinfection with the phage does not result in any detectable increase in the rate of phage production by the lysogenic culture. Phage $\beta$ provides a striking exception to the rule of immunity, for superinfection of strain Davis $(\beta)$ with phage $\beta$ causes a considerable increase in phage production. Generally, too, a lysogenic strain does not differ from its non-lysogenic relative in colonial appearance. The colonial appearance of strain Davis, which absorbs phage $\beta$ rapidly, was grossly altered by lysogenicity with phage $\beta$, presumably because of its incomplete immunity. Dr Six (personal communication) has similarly found that in the case of the Shigella shigae phage $\boldsymbol{P}_{2}$, the immunity of a lysogenic strain is not absolutely complete, for about one organism in $10^{-3}$ superinfected will lyse as a consequence of superinfection.

\section{REFERENCES}

Bertani, G. (1951). Studies on lysogenesis. I. The mode of phage liberation by lysogenic Escherichia coli. J. Bact. 62, 293.

Bertani, G. (1953). Lysogenic versus lytic cycle of phage multiplication. Cold Spr. Harb. Symp. quant. Biol. 18, 65.

Bertani, G. (1954). Studies on lysogenesis. III. Superinfection of lysogenic Shigella dysenteriae with temperate mutants of the carried phage. J. Bact. 67, 696 .

Bertani, G. (1958). Lysogeny. Advanc. Virus Res. 5 (in the Press).

Bordet, J. \& Renaux, E. (1930). L'influence du calcium sur l'évolution des cultures de Charbon. Ann. Inst. Pasteur, 45, 1.

Boyd, J. S. K. (1951). Observations on the relationship of symbiotic and lytic bacteriophage. J. Path. Bact. 63, 445.

Boyd, J. S. K. (1954). Bacteriophage and heredity. Nature, Lond. 173, 1050.

Bradley, P. L. \& Boyd, J. S. K. (1952). Readsorption of phage produced in cultures of lysogenic strains of Salmonella typhimurium. J. Path. Bact. 64, 891.

Burnet, F. M. \& Lush, D. (1936). Induced lysogenicity and mutation of bacteriophage within lysogenic bacteria. Aust. J. exp. Biol. med. Sci. 14, 27. 
CHU, H. P. (1949). A comparative study of Bacillus anthracis and its related organisms. Ph.D. Thesis, University of Cambridge.

CHu, H. P. (1952). Variation of Bacillus anthracis with special reference to the noncapsulated avirulent variant. J. Hyg., Camb. 50, 433.

Clarke, N. A. (1952). Studies on the host-virus relationship in a lysogenic strain of Bacillus megatherium. II. The growth of Bacillus megatherium in synthetic medium. J. Bact. 63, 187.

FIsk, R. T. (1942). Studies on Staphylococci. I. Occurrence of bacteriophage carriers among strains of Staphylococcus aureus. J. infect. Dis. 71, 153.

Hershey, A. D., Kalmanson, G. \& Bronfenbrenner, J. (1943). Quantitative methods in the study of the phage-antiphage reaction. J. Immunol. 46, 267.

Ionesco, H. (1953). Sur une propriété de Bacillus megaterium liée à la présence d'un prophage. C.R. Acad. Sci., Paris, 237, 1794.

Jacob, F., Lwoff, A., Srminovitch, L. \& Wollman, E. L. (1953). Définition de quelques termes relatifs à la lysogénie. Ann. Inst. Pasteur, 84, 222.

$\mathrm{J}_{\mathrm{ACOB}}, \mathrm{F}$. \& Woliman, E. L. (1953). Induction of phage development in lysogenic bacteria. Cold Spr. Harb. Symp. quant. Biol. 18, 101.

JACOB, F. \& Wollman, E. L. (1954). Étude génétique d'un bactériophage tempéré d'Escherichia coli. I. Le systeme génétique du bacteriophage $\lambda$. Ann. Inst. Pasteur, 87, 653.

Kaiser, A. D. (1955). A genetic study of the temperate coliphage $\lambda$. Virology, 1, 424.

KNight, B. C. J. G. \& Proom, H. (1950). A comparative survey of the nutrition and physiology of mesophilic species in the genus Bacillus. J. gen. Microbiol. 4, 508.

LwoFf, A. (1953). Lysogeny. Bact. Rev. 17, 269.

McCloy, E. W. (1951). Studies on a lysogenic Bacillus strain. I. A bacteriophage specific for Bacillus anthracis. J. Hyg., Camb. 49, 114.

MCCloy, E. W. (1953). Sporulation of bacteria despite infection by virulent phage. VI. Congr. int. Microbiol. 2, 210.

Mires, A. A. \& Misra, S. S. (1938). The estimation of the bactericidal power of the blood. J. Hyg., Camb. 38, 732.

Renaux, E. (1952). Culture de Bacillus anthracis en milieu calcique et en milieu oxalate. Ann. Inst. Pasteur, 83, 38.

Scholtens, R. Th. (1950). Lysogenicity as a tool for bacteriophage typing of Salmonella paratyphi B. Leeuwenhoek ned Tijdschr. 16, 256.

Smith, N. R., Gordon, R. E. \& Clark, F. E. (1952). Aerobic Sporeforming Bacteria. U.S. Department of Agriculture. Agriculture monograph No. 16.

Sterne, M. (1937). Variation in Bacillus anthracis. Onderstepoort J. vet. Sci. 8, 271.

Swanstrom, M. \& Adams, M. H. (1951). Agar layer method for production of high titre phage stocks. Proc. Soc. exp. Biol., N.Y. 78, 372.

Twont, F. W. (1915). An investigation on the nature of ultra-microscopic viruses. Lancet, ii, 1241.

Weigle, J. J. (1953). Induction of mutations in a bacterial virus. Proc. nat. Acad. Sci., Wash. 39, 628.

Weinberg, E. D. (1955). The effect of $\mathrm{Mn}^{++}$and antimicrobial drugs on sporulation of Bacillus subtilis in nutrient broth. J. Bact. 70, 289.

\section{EXPLANATION OF PLATE}

Fig. 1. Plaques of phage $\alpha$ and phage $\beta$ on strain Davis.

Fig. 2. Plaques of phage $\beta \mathrm{LC}$ on strain Davis.

Fig. 3. Colonies of non-lysogenic Davis.

Fig. 4. Colonies of Davis $(\beta)$.

Fig. 5. Colonies of Davis $(\beta \mathrm{LC})$.

Fig. 6. Plaques produced by $\gamma$-infected bacteria of non-lysogenic Davis on Davis $(\beta \mathrm{LC})$. 
Journal of General Microbiology, Vol. 18, No. 1
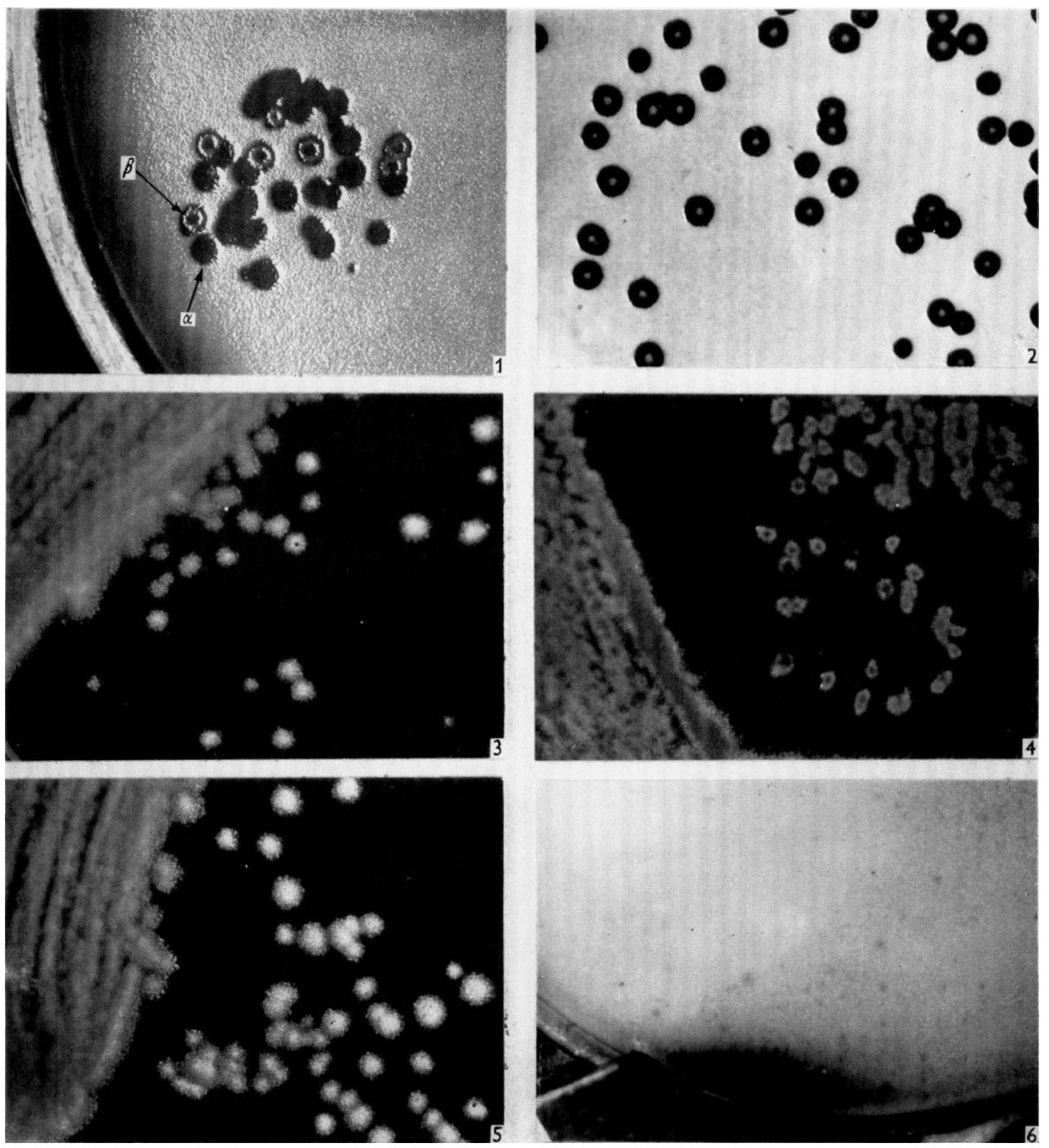

E. W. McCloy-Lysogenicity and immunity. Plate 1 